\title{
Visuoconstructional Impairment in Dementia Syndromes
}

\author{
WILLIAM E. REICHMAN, ${ }^{1}$ JEFFREY L. CUMMINGS, ${ }^{2}$ \\ KEITH D. MaDANIEL, ${ }^{2}$ FREDERICK FLYNN ${ }^{2}$ and JEFFREY GORNBEIN ${ }^{2}$ \\ ${ }^{1}$ COPSA Institute for Alzheimer's Disease and Related Disorders, Community Mental Health Center at \\ Piscataway, New Jersey \\ Department of Psychiatry, Robert Wood Johnson Medical School, University of Medicine and \\ Dentistry of New Jersey, Piscataway, New Jersey \\ ${ }^{2}$ Neurobehavior Unit, West Los Angeles VAMC (Brentwood Division), California \\ Departments of Neurology, Psychiatry $\&$ Biobehavioral Sciences, and Biomathematics, UCLA School \\ of Medicine, Los Angeles, California
}

Correspondence: William E. Reichman, M.D., Medical Director, COPSA Institute for Alzheimer's Disease and Related Disorders, University of Medicine and Dentistry of New Jersey, 667 Hoes Lane, Piscataway, New - Fersey 08855-1392, USA

\begin{abstract}
Dementia of the Alzheimer type (DAT) affects most neuropsychological domains including language, memory, and visuo-spatial skills. The latter are usually assessed by poorly quantifiable copying tasks. We assessed constructional abilities using the Developmental Test of Visuomotor Integration (VMI) comprised of a series of model drawings of increasing complexity. Twenty-six patients meeting NINCDS-ADRDA criteria for DAT, 21 normal aged subjects with normal mental status examinations, and 14 patients with vascular dementia were tested. In DAT, we found significant correlations between visuoconstructive ability and memory registration, delayed recall, and language functions such as confrontation naming and word-list generation. Less marked, but significant correlations were found in the vascular dementia group between visuoconstructive ability and memory registration and word-list generation. A few normal elderly subjects were unable to copy the most challenging figures. The study demonstrates that: (1) VMI is a convenient method for quantifying constructional deficits in DAT and other dementing illnesses; (2) constructional deficits are highly correlated with dementia severity and memory and language deficits in DAT; (3) neuropsychological deficits are less highly inter-correlated in vascular dementia than in DAT; and (4) abnormal constructional skills are present in some normal elderly.
\end{abstract}

\section{Introduction}

Dementia of the Alzheimer type (DAT) accounts for approximately 50$60 \%$ of all causes of acquired intellectual decline while vascular etiologies account for 13-25\% of dementias (Clarfield, 1988). The neuropsychological characteristics of DAT have been well described. They include disturbances in language, memory, cognition (calculation, abstraction, problem solving), personality and mood, and visuo-spatial function (Cummings et al., 1986; Becker et al., 1988). Early in the course of DAT, visuospatial deficits become apparent as patients exhibit environmental disorientation and have diffi-

$0953-4180 / 91 / 030153 \times 10 \$ 3.50 / 0 \quad$ (C) 1991 CNS (Clinical Neuroscience) Publishers 
culty dressing (Henderson et al., 1989). On neuropsychological testing, such patients demonstrate impaired performance on tasks that require the copying of figures (visuoconstructive ability) (Henderson et al., 1989; Brouwers et al., 1984). In DAT, these deficits are thought to reflect the characteristic pathologic involvement of parietal cortices by neuronal loss, neuritic plaques, neurofibrillary tangles, amyloid angiopathy, and deficits in acetylcholine and other essential neurotransmitters (Brouwers et al., 1984).

In vascular dementia, acquired deficits also involve multiple intellectual functions often including disturbances in visuoconstructive ability. Focal pathology involving the right parietal lobe is particularly likely to produce visuospatial deficits (Mesulam, 1981). Lesions involving frontal systems may also result in impaired planning, sequencing and strategy, contributing to visuoconstructional disturbances (Stuss and Benson, 1986). The neuropsychological syndrome of vascular dementia has been characterized as "patchy" with involved and spared functions varying from patient to patient (APA, 1987). No operationalized criteria have been developed for this concept.

In most studies, visuoconstructive abilities are assessed by having patients draw or copy relatively complex figures such as houses, flowers, or clocks. These figures are sensitive to the deficits of demented patients but are difficult to quantify and do not facilitate investigation of the entire spectrum of constructional abnormalities.

We initiated the current study to examine performance on a readily quantifiable visuoconstructive task, the Developmental Test of VisualMotor Integration (VMI) (Beery, 1989) and to seek correlations between visuoconstructive ability and performance on tasks assessing memory and language in patients with well-defined DAT. We then compared these results with two additional groups of subjects: patients with vascular dementia and healthy non-demented older adults. We hypothesized that both dementia groups would perform significantly more poorly than nondemented subjects on the VMI and that visuoconstructive deficits in the DAT group would correlate strongly with impairment in memory, language, and overall dementia severity. We posited that while such correlations might also exist in the vascular dementia patients, they would be less uniform than in DAT. We further hypothesized that "patchiness" of deficits is expressed as low intercorrelations between scores on tests of different intellectual functions.

\section{Subjects}

Two patient groups (DAT and vascular dementia) and an age-matched non-demented control group were studied. All patients underwent physical, neurological, and mental status examinations and had a thorough laboratory evaluation, electrocardiogram, chest radiograph, and computerized tomography (CT) or magnetic resonance imaging (MRI) of the head. The DAT group was comprised of 26 patients who met criteria for "probable Alzheimer's disease" as defined by the National Institute of Neurological 
Disorders and Stroke-Alzheimer's Disease and Related Disorders Association Work Group (McKhann et al., 1984). The vascular dementia group was comprised of 14 patients who had Hachinski Ischemia Scale Scores (Hachinski et al., 1975) greater than 7 and who met criteria for multi-infarct dementia as outlined in the revised third edition of the Diagnostic and Statistical Manual of Mental Disorders (DSM-IIIR) (APA, 1987). The control group was composed of 21 healthy, volunteer subjects who selfreported no history of intellectual deficits or significant neurologic or psychiatric illness. All control subjects had Mini-Mental State Examination (MMSE) (Folstein et al., 1975) scores of at least 27 out of a possible 30. There was no significant difference in age among the three groups (overall mean $=71$ years, range $47-88$ years). The DAT group had significantly more years of formal education than the controls (mean difference $=2.3$ years, $p<0 \cdot 05)$.

\section{Methods}

A comprehensive battery of neuropsychological tests assessing visuoconstructive ability, memory, language, and overall dementia severity was administered to each study participant. Visuoconstructive ability was measured by performance on the VMI (0-24 points). Following standard instructions, subjects were asked to copy a series of 24 increasingly complicated figures. The figures to be copied range from a simple straight line (VMI item \#1) to complex interdigitating stars (VMI item \#24). Each reproduced figure was then scored by the examiner as pass or fail using the test's detailed standardized criteria. Total score on the VMI reflected the number of copied figures that were passed. For individuals aged 14 years and above a score of 18 out of a possible 24 represents two standard deviations below the mean. The VMI has been used extensively in the study of visuoconstructive development in children and has proven to be reliable and valid (Beery, 1989).

We assessed memory function by administering the Shopping-list test (McCarthy et al., 1981) modified by shortening the list of words to be learned from 10 to 5 . There were three separate component scores to this task: memory registration, delayed spontaneous recall, and delayed recall with cueing. Subjects were read a list of five grocery items and asked to repeat them over several trials until all five items were learned, or a total of five trials were completed, whichever came first. The memory registration score reflected the total number $(0-5)$ of items learned on the last completed trial prior to a 15 min delay. The delayed spontaneous recall score $(0-5)$ represented the total number of items spontaneously recalled by the subject after a 15 min delay. The delayed recall with cueing score (0-5) represented the delayed spontaneous recall score plus any additional items remembered after categorical and multiple choice cues were provided.

Language integrity was measured by performance on the Boston Naming Test (Kaplan et al., 1982), a test of confrontation naming (0-60 points), and word-list generation (total number of animals generated by the patient 
in $1 \mathrm{~min}$ ) (Goodglass and Kaplan, 1983), a test of verbal fluency. Overall dementia severity was measured by the MMSE.

\section{Statistical analysis}

Associations between VMI scores and measures of memory, language and dementia severity were assessed using the Spearman (rank) correlation. This assessment was done separately for the three groups of subjects (DAT, vascular dementia, normals). Both the simple correlations and partial correlations controlling for severity (as measured by MMSE) were computed.

Mean VMI, memory, language and dementia severity scores across the three groups were compared using ANOVA and post-hoc t-tests. The standard errors used in these t-tests were based on the pooled variance across the three groups (one-way ANOVA, within-group pooled variance). The Tukey (studentized range) significance criterion was used to adjust for multiple t-tests so that the overall type I error (alpha level) was 0.05 or less.

Since we suggested that the relationship of VMI to memory and language might be affected by overall dementia severity, VMI scores in DAT and vascular dementia patients were compared after stratifying into high versus low MMSE groups using an MMSE cutoff score of 15.

\section{Results}

Table 1 demonstrates the means and ranges of scores obtained for all three groups of subjects on the neuropsychological tests administered. In the DAT group, MMSE scores ranged from $0-26($ mean $=10)$. This was significantly lower than the controls (mean difference $=18 \cdot 0, p<0.05$ ) and the vascular dementia group (mean difference $=8 \cdot 1 . p<0 \cdot 05$ ) (Table 2). Representative examples of impaired visuoconstructive ability in two DAT subjects is illustrated in Fig. 1. The mean VMI for the entire DAT group was 8 with a performance range of 0 to 23 .

Correlational analyses of performance on each individual neuropsychological test with VMI score is shown for all three groups of subjects in Table 3. In the DAT group, VMI scores strongly correlated with MMSE performance $(r=0.91, \quad p=0.0001)$, word-list generation $(r=0.91$, $p=0.0001)$, Boston Naming Test $(r=0 \cdot 85, p=0.0001)$, and memory registration and delayed recall with cueing sections of the modified Shopping List Test $(r=0.81, p=0.0001 ; r=0.66, p=0.001$ respectively). There was a significant but less marked correlation between VMI and the delayed spontaneous recall section of this memory test $(r=0.47, p<0.02)$.

In the vascular dementia group, VMI scores ranged from 1 to 23 (mean score 13). Significant, but less compelling relationships were observed between VMI scores and word-list generation $(r=0.58, p<0.05)$ and memory registration $(r=0.78, p<0.005)$. No other correlations emerged.

For the control group, VMI scores ranged from a low of 15 to a high of 24 (perfect score). Significant correlations were noted between VMI and 
TABLE 1. Characteristics and neuropsychological performance of dementia patients and control subjects (mean and range)

\begin{tabular}{lccc}
\hline & $\begin{array}{c}D A T \\
(\mathcal{N}=26)\end{array}$ & $\begin{array}{c}\text { Vascular dementia } \\
(\mathcal{N}=14)\end{array}$ & $\begin{array}{c}\text { Controls } \\
(\mathcal{N}=21)\end{array}$ \\
\hline Age (yrs) & $72(52-85)$ & $70(47-80)$ & $71(50-88)$ \\
Education (yrs) & $14(9-16)$ & $12(8-16)$ & $12(8-16)$ \\
MMSE & $10(0-26)$ & $18(10-29)$ & $28(27-30)$ \\
VMI & $8(0-23)$ & $13(1-23)$ & $21(15-24)$ \\
Word-list & $4(0-14)$ & $8(1-21)$ & $20(8-48)$ \\
Boston Naming Test & $15(0-56)$ & $35(4-53)$ & $49(17-60)$ \\
$\begin{array}{l}\text { Memory-regist. } \\
\text { Memory-delayed spontaneous }\end{array} \quad 2(0-5)$ & $4(1-5)$ & $5(5)$ \\
$\quad$ recall & $0(0-3)$ & $1(0-4)$ & $4(1-5)$ \\
Memory-delayed recall +cueing & $1(0-4)$ & $3(0-5)$ & $5(4-9)$ \\
\hline
\end{tabular}

DAT indicates dementia of the Alzheimer type; MMSE, Mini-Mental State Examination; and VMI, Developmental Test of Visuomotor Integration. Values are expressed as mean, with range in parenthesis.

TABLE 2. Differences between means for all groups of subjects ( $t$-test comparisons)

\begin{tabular}{lccc}
\hline & $\begin{array}{c}D A T \text { vs } \\
\text { controls }\end{array}$ & $\begin{array}{c}\text { Vascular dementia } \\
\text { vs controls }\end{array}$ & $\begin{array}{c}\text { DAT vs } \\
\text { vascular } \\
\text { dementia }\end{array}$ \\
\hline Age (yrs) & $0 \cdot 6$ & $1 \cdot 1$ & $1 \cdot 6$ \\
Education (yrs) & $2 \cdot 3^{*}$ & $0 \cdot 4$ & $1 \cdot 9$ \\
MMSE & $18 \cdot 0^{*}$ & $9 \cdot 9^{*}$ & $8 \cdot 1^{*}$ \\
VMI & $12 \cdot 8^{*}$ & $8 \cdot 1^{*}$ & $4 \cdot 7$ \\
Word-list & $16 \cdot 1^{*}$ & $12 \cdot 0^{*}$ & $4 \cdot 1$ \\
Boston Naming Test & $34 \cdot 1^{*}$ & $13 \cdot 9^{*}$ & $20 \cdot 2^{*}$ \\
Memory-registration & $3 \cdot 3^{*}$ & $0 \cdot 6$ & $2 \cdot 6^{*}$ \\
Memory-delayed spontaneous recall & $3 \cdot 3^{*}$ & $2 \cdot 7^{*}$ & $0 \cdot 6$ \\
Memory-delayed recall+cueing & $4 \cdot 2^{*}$ & $2 \cdot 6^{*}$ & $1 \cdot 6^{*}$ \\
\hline
\end{tabular}

DAT indicates dementia of the Alzheimer type; MMSE, Mini-Mental State Examination; VMI, Developmental Test of Visuomotor Integration. Values are expressed as difference between means. ${ }^{*} p<0.05$

MMSE scores $(r=0.53, p<0.02)$, word-list generation $(r=0.59, p<0.005)$, and Boston Naming Test scores $(r=0.65, p<0.005)$.

DAT patients were significantly more severely intellectually impaired, as measured by the MMSE, than the vascular dementia patients, and differences between the VMI performances of the two groups might be attributed to dementia severity. Within the vascular dementia patients, however, there was no correlation between VMI and MMSE scores $(r=0.33$, unadjusted $p=0.25)$, whereas in DAT there was a strong relationship between VMI and MMSE $(r=0.91, p=0.0001$, Tukey $p<0.02)$. Thus, in the vascular dementia group, constructional disturbances cannot be attributed to overall severity of dementia (MMSE score); in the DAT group the two were strongly associated. A similar independence of MMSE and 

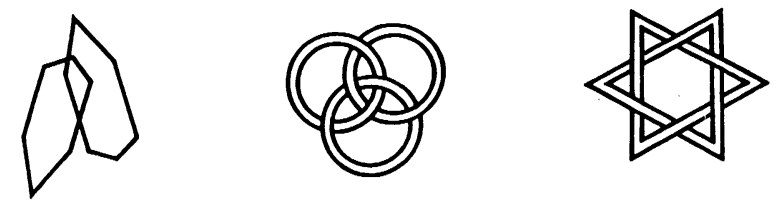

patient 1 $\begin{aligned} V M I & =15 \\ M M S E & =11\end{aligned}$
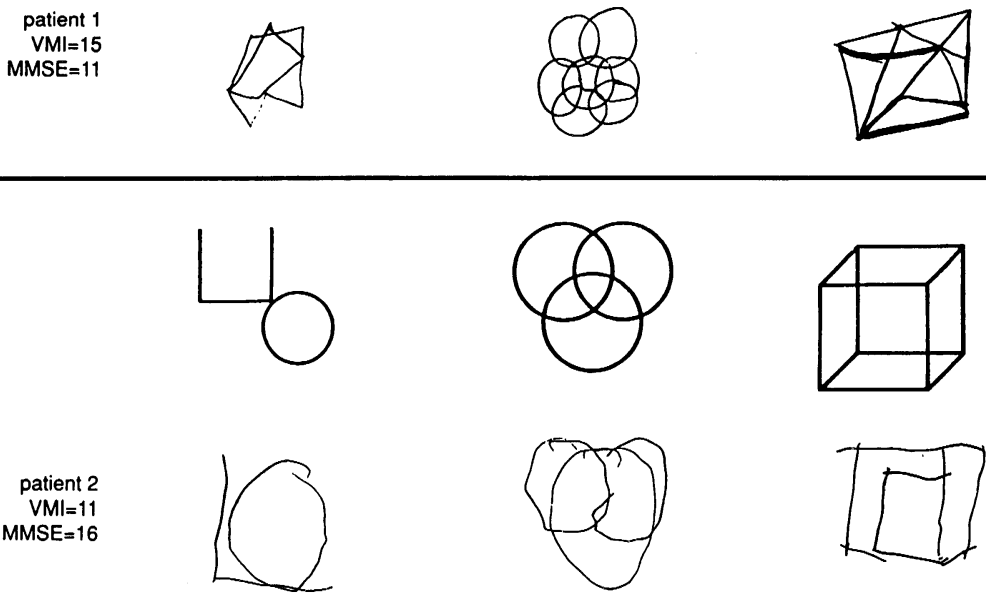

NOTE: upper rows show VMl test figures.

FIG. 1. Representative examples of visuoconstructive deficits on the Development Test of Visuomotor Integration from two patients with dementia of the Alzheimer type.

TABLE 3. Correlational analyses of test variables with VMI ( $r$ values)

\begin{tabular}{lccc}
\hline & $D A T$ & Vascular dementia & Controls \\
\hline Age (yrs) & 0.02 & -0.26 & -0.05 \\
Education (yrs) & -0.10 & 0.21 & -0.02 \\
MMSE & $0.91_{\ddagger}^{\ddagger}$ & 0.33 & $0.53^{*}$ \\
Word-list & $0.91_{\ddagger}^{\ddagger}$ & $0.58^{* *}$ & $0.59 \dagger$ \\
Boston Naming Test & $0.85_{\ddagger}^{\ddagger}$ & 0.28 & $0.65 \dagger$ \\
Memory-regist. & $0.81_{\ddagger}^{\ddagger}$ & $0.78 \dagger$ & - \\
Memory-delayed spontaneous recall & $0.47^{*}$ & -0.12 & 0.42 \\
Memory-delayed recall +cueing & $0.66_{\ddagger}^{\ddagger}$ & 0.26 & -0.06
\end{tabular}

DAT indicates dementia of the Alzheimer type; MMSE, Mini-Mental State Examination; and VMI, Developmental Test of Visuomotor Integration. Listed values represent Spearman rank correlation coefficients. Tukey $p$ values: ${ }^{*} p<0.02, * * p<0.05, \dagger p<0.005,{ }_{\ddagger} p=0.0001$

performance on memory tests (registration, spontaneous recall, recall with cueing) and the Boston Naming Test was evident in the vascular dementia group; all these scores were significantly correlated with the MMSE in DAT. The overall pattern indicated that performances were not closely interrelated in vascular dementia whereas in DAT, skills in individual domains were strongly associated with the magnitude of overall cognitive decline and with each other. 
Pass or fail performance on the construction item of the MMSE (overlapping pentagons) was compared with VMI scores for both the DAT and vascular dementia groups. In both groups of patients, no individuals with VMI scores below 13 were able to pass the MMSE construction. Three out of 21 control subjects failed the MMSE construction.

\section{Discussion}

The present study demonstrates that the VMI is an easily administered and readily quantifiable test of visuoconstructive competence that accurately distinguishes demented patients from normal controls. We confirmed that while impairment on visuo-constructional tasks is a common finding in DAT patients, the severity of impairment varies considerably. Moreover, in DAT, deficits in visuoconstructive ability closely parallel impairment in other intellectual functions such as memory, language, and overall dementia severity. The VMI lends itself to assessing severely impaired patients with marked behavioral disturbances and disorientation as well as relatively intact patients whose deficits can be revealed only by complex constructional tasks.

In the vascular dementia patients, deficits in visuoconstructive ability correlated best with deficits in word-list generation, and impairment in memory registration. The more modest strength of these relationships compared to DAT and the lack of significant correlation between visuoconstructive ability and performance on tests of confrontation naming, recent memory, and overall dementia severity provide corroborative evidence that deficits in vascular dementia are less predictable than in DAT. We suggest that one potential definition for "patchy" deficits in vascular dementia is that neuropsychological deficits are not highly intercorrelated.

Within our control group of aged normal individuals the mean VMI score was 21 out of a possible 24. Performance was variable (range 15-24) although no control subjects scored less than two standard deviations below the mean. Fourteen percent of the controls however, failed the MMSE construction. These data suggest that in our non-demented elderly control population, some individuals had difficulty copying figures. Design constraints make it unlikely that visual, motor, or psychomotor speed deficits would account for the observed abnormalities. Several possible explanations for the variable performance can be proferred. First, a number of our controls are in the initial phases of a dementing illness. Impaired performance on the VMI may be a prelude to the development of further intellectual decline. Second, visuoconstructive skills may be variable in the cohort and such performances would have been evident had they been tested in youth. Finally, decline in performance on demanding visuospatial tasks may be a feature of normal aging. Albert and Kaplan (1980) analyzed the visuospatial abilities of a large number of individuals included in a normative aging study who were tested with simple and complex constructions and object assembly tasks. They concluded from the error patterns that the constructional performance of normal elderly individuals is character- 
ized by a tendency to misplace or reorient stimulus details, segment the elements of the model, and isolate and focus on the most visually salient details. They hypothesized that such errors represented declining integrity of frontal systems in the course of normal aging. Similar types of errors were evident in the performance of our non-demented aged subjects, supporting the hypothesis that such changes may be evident in the normal elderly.

Visuospatial and constructional deficits have been the subject of relatively few studies in DAT. Moore and Wyke (1984) compared 15 subjects with DAT and 15 elderly control subjects and found that DAT patients did significantly worse than controls on both the drawing and copying task. There were significant correlations between dementia severity as reflected on a rating scale and on several subtests of an intelligence test and the constructional performances. They noted that the drawings of DAT patients had both the impoverishment of detail previously reported in patients with left brain lesions and the fragmentation observed in the productions of patients with right brain lesions. More recently, Henderson and colleagues (1989) studied spontaneous drawings and copies of houses and clocks by 28 DAT patients; significant correlations were evident between constructional abilities and the MMSE, a spouse-rated spatial disorientation index, and the Boston Naming Test. Similarly, de Leon et al. (1984) found that DAT patients who wandered were significantly more impaired on a battery of visuospatial tests than those who did not exhibit this behavior. These studies indicate that constructional deficits are common in DAT and they may be important contributors to behavioral disorders such as wandering.

Among our DAT patients, there was only one subject whose cognitive performance reflected asymmetric hemispheric involvement. This subject's visuoconstructive ability was only minimally impaired $(V M I=20)$ while his language function, as tested by confrontation naming and verbal fluency, was significantly deficient (Boston Naming Test $=12$, word-list generation =9). Foster et al. (1983) and Celsis and co-workers (1987) have reported significant numbers of DAT patients with asymmetric clinical features. In our series of DAT patients, however, with the exception of the one subject cited above, disturbances of visuoconstructive function was regularly related to deficits in language and memory.

The pathophysiologic correlates of constructional deficits in DAT have been explored using positron emission tomography (PET), CT, cholinergic treatment, and neuropsychological evaluations. PET studies reveal that the performance subtests of the Wechsler Adult Intelligence Scale (WAIS) (Wechsler, 1981) are more highly correlated with right parietal glucose hypometabolism, suggesting that constructional abnormalities are more determined by right brain dysfunction in DAT (Foster et al., 1983; Celsis et al., 1987; Haxby et al., 1985). Significant correlations have also been found between visuospatial tests and cerebral atrophy in DAT as reflected in the width of the lateral ventricular bodies $(r=-0 \cdot 63)$, width of the frontal horns $(r=-0.07)$, and width of the third ventricle $(r=-0.49)$ (Eslinger $e t$ al., 1984). Experimental physostigmine treatment of DAT resulted in a 
significant improvement in constructional skills with greater effect on constructions than memory (Muramoto et al., 1984). The response suggests that the marked cholinergic deficits of DAT contribute importantly to the constructional abnormalities. Together, the existing evidence suggests that constructional disturbances in DAT reflect cortical degenerative and biochemical alterations and that posterior right hemisphere dysfunction may be most involved in production of these deficits.

In conclusion, our data demonstrate that in DAT, visuoconstructive deficits, as measured by the VMI, correlate well with deficits in other intellectual spheres such as memory and language. In patients with vascular dementia, significant correlations exist between VMI scores and memory registration and verbal fluency. The pattern relating visuospatial impairment to other deficits is different in the two disorders. In a subset of identified healthy, non-demented subjects, performance on the more complex items of the VMI may be impaired.

\section{Acknowledgement}

The authors wish to extend their gratitude to Ms Ann Rauchbach for her patience and care in the preparation of this manuscript.

\section{References}

Albert, M. S. and Kaplan, E. F. (1980). Organic implications of neuropsychological deficits in the elderly. In "New Directions in Memory and Aging, Proceedings of the George A. Talland Memorial Conference", (Eds L. W. Poon, J. Fozard, L. Cermak, D. Arenberg and L. W. Thompson). Lawrence Erlbaum, pp. 403-432.

American Psychiatric Association (1987). Diagnostic and Statistical Manual of Mental Disorders, 3rd Edn, Revised. American Psychiatric Association, Washington, D.C.

Becker, J. T., Huff, J., Nebes, R. D., et al. (1988). Neuropsychological function in Alzheimer's disease: pattern of impairment and rates of progression. Archives of Neurology, 45, 263268.

Beery, K. E. (1989). "Developmental Test For Visual-Motor Integration”. Modern Curriculum Press, Cleveland.

Brouwers, P., Cox, C., Martin, A., et al. (1984). Differential perceptual-spatial disorientation in Huntington's and Alzheimer's dementias. Archives of Neurology, 41, 1073-1076.

Celsis, P., Agniel, A., Puel, M., et al. (1987). Focal cerebral hypoperfusion and selective cognitive deficit in dementia of the Alzheimer type. Journal of Neurology, Neurosurgery and Psychiatry, 50, 1602-1612.

Clarfield, A. M. (1988). The reversible dementias: do they reverse? Annals of Internal Medicine, 109, 476-486.

Cummings, J. L. and Benson D. F. (1986). Dementia of the Alzheimer type: an inventory of diagnostic clinical features. $\mathcal{F} A G S, 34,12-19$.

de Leon, M. J., Potegal M. and Gurland, B. (1984). Wandering and parietal signs in senile dementia of Alzheimer's type. Neuropsychobiology, 11, 155-157.

Eslinger, P.J., Damasio H., Graff-Radford, N., et al. (1984). Examining the relationship between computed tomography and neuropsychological measures in normal and demented elderly. Fournal of Neurology, Neurosurgery and Psychiatry, 47, 1319-1325.

Folstein, M. F., Folstein, S. E. and McHugh, P. R. (1975). "Mini-mental" state": a practical method for grading the cognitive state of patients for the clinician. Fournal of Psychiatric Research, 12, 189-198. 
Foster, N. L., Chase, T. N., Fedio, P., et al. (1983). Alzheimer's disease: focal cortical changes shown by positron emission tomography. Neurology, 33, 961-965.

Goodglass, H. and Kaplan, E. (1983). "The Assessment of Aphasia and Related Disorders", 3rd Edn. Lea \& Febiger, Philadelphia.

Haxby, J. V., Duara, R., Grady, C. L., et al. (1985). Relations between neuropsychological and cerebral metabolic asymmetries in early Alzheimer's disease. Fournal of Cerebral Blood Flow Metabolism, 5, 193-200.

Hachinski, V. C., Iliff, L. D., Zilhka, L., et al. (1975). Cerebral blood flow in dementia. Archives of Neurology, 32, 632-637.

Henderson, V.W., Mack, W. and Williams, B. W. (1989). Spatial disorientation in Alzheimer's disease. Archives of Neurology, 46, 391-394.

Kaplan, E., Goodglass, H. and Weintraub, S. (1982). "Boston Naming Test". Lea \& Febiger, Philadelphia.

McCarthy, M., Ferris, S. H., Clark, E., et al. (1981). Acquisition and retention of categorized material in normal aging and senile dementia. Experimental Aging Research, 7, 127-135.

McKhann, G., Drachmann, D., Folstein, M., et al. (1984). Clinical diagnosis of Alzheimer's disease: report of the NINCDS-ADRDA Work Group under the auspices of Department of Health and Human Services Task Force on Alzheimer's Disease. Neurology, 34, 939-944.

Mesulam, M. M. (1981). A cortical network for directed attention and unilateral neglect. Archives of Neurology, 10, 309-325.

Moore, V. and Wyke, M. A. (1984). Drawing disability in patients with senile dementia. Psychological Medicine, 14, 97-105.

Muramoto, O., Sugishita, M. and Ando, K. (1984). Cholinergic system and constructional praxis: a further study of physostigmine in Alzheimer's disease. Fournal of Neurology, Neurosurgery and Psychiatry, 47, 485-491.

Stuss, D. T. and Benson, D. F. (1986). "The Frontal Lobes". Raven Press, New York, p. 158.

Wechsler, D. (1981). "Manual for the Wechsler Adult Intelligence Scale": Revised. Psychological Corp., New York. 


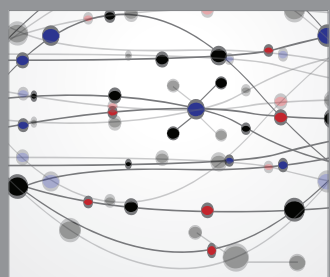

The Scientific World Journal
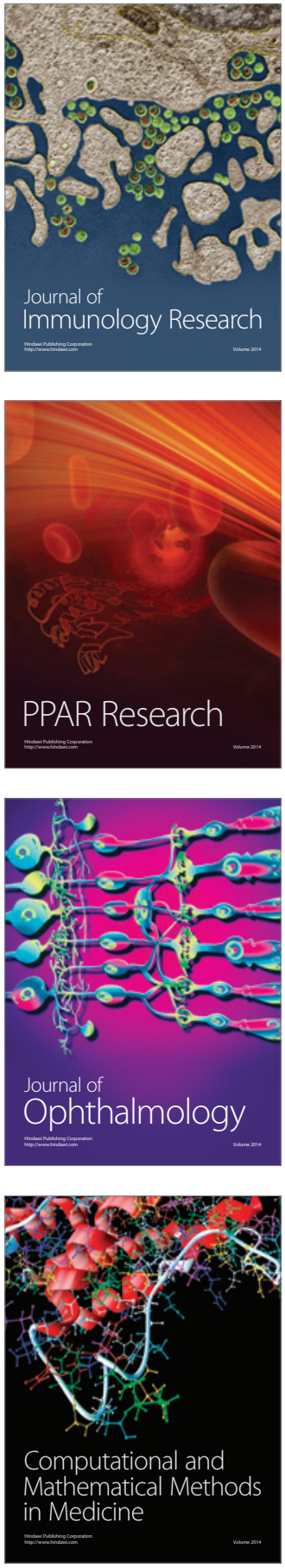

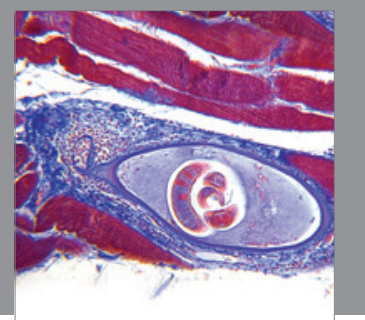

Gastroenterology

Research and Practice
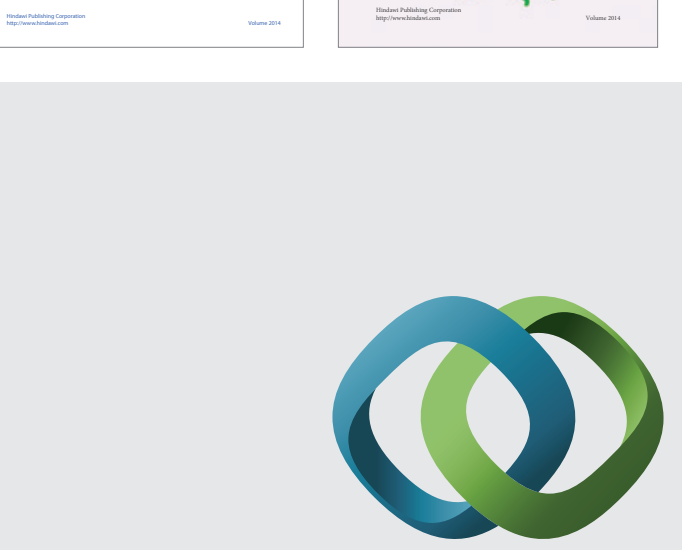

\section{Hindawi}

Submit your manuscripts at

http://www.hindawi.com
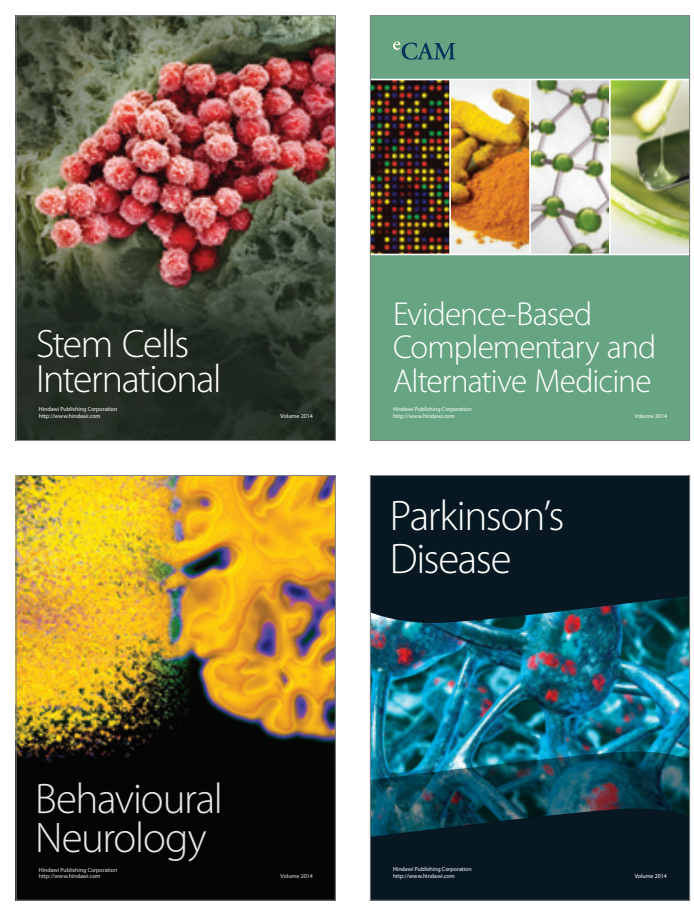

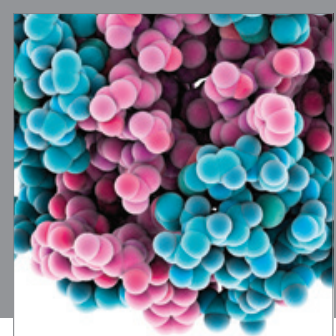

Journal of
Diabetes Research

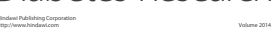

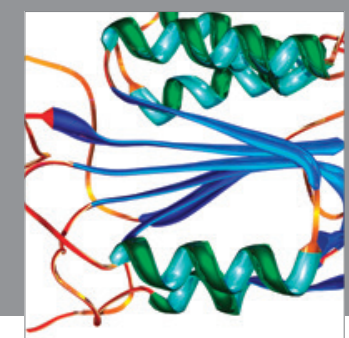

Disease Markers
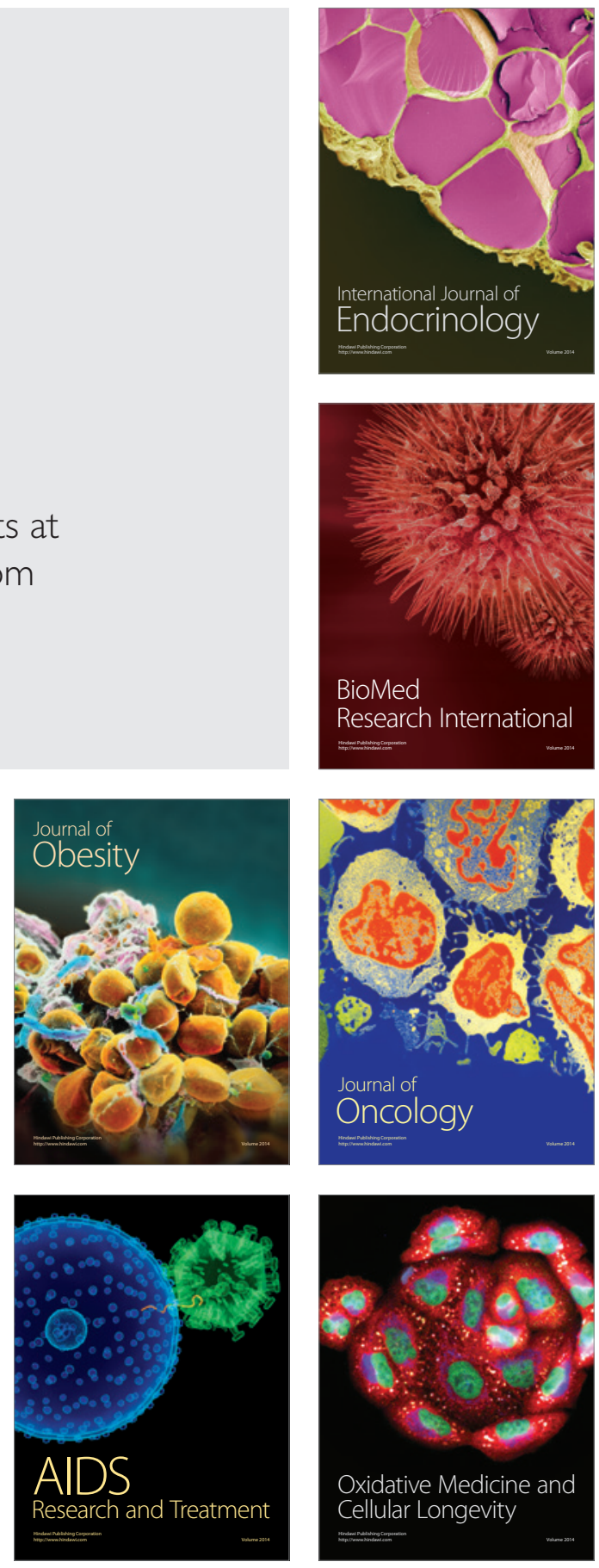